\title{
Inhibition of methanogenesis by human bile
}

\author{
T H J Florin, H J Woods
}

\begin{abstract}
The factors that regulate methanogenesis in humans have not been established. The presence of bile acid, which is lost into the colon from the small intestine, may be an important regulatory factor of methanogenesis. To examine this possibility, the effect of human bile on methane production by faecal cultures, and the in vivo effect of biliary diversion on breath methane excretion in a methanogenic choledochostomy patient, were investigated. Faecal suspensions $(0 \cdot 1 \%)$ from five methanogenic humans were incubated anaerobically with bile (0.3-30\%) from three choledochostomy patients, and headspace methane measured by gas chromatography. All biles inhibited headspace methane. Inhibition of methanogenesis was dose dependent, plateaued at $10-30 \%$ bile concentration, and was abolished by $0.6 \%$ cholestyramine. The maximum inhibition by bile, median (range), was $38(0.9-56) \%$ of control methane values. Reversal of the bile fistula in the fourth choledochostomy patient converted that subject from methanogenic to 'non-methanogenic' status. It is concluded that inhibition of methanogens in the caecum by bile acid could significantly reduce the number of methanogens in the colon. This and the effect of transit time could explain much of the known epidemiology of 'nonmethanogenesis', which has been related to obesity, (comparatively) fast colonic transit in healthy persons, and to small intestinal Crohn's disease.

(Gut 1995; 37: 418-421)
\end{abstract}

Keywords: bile, human, methane, methanogenesis, methanogens.

Production of methane gas (methanogenesis) by colonic flora may be important for several reasons. Firstly, it permits a more complete fermentation of 'fibre' because the removal of hydrogen is energetically more favourable for fermentation. ${ }^{1}$ The clinical relevance of this is not known. The prevalence of methanogenesis, however, is inversely related to the prevalence of bowel cancer ${ }^{2}$ in contrasting African populations. ${ }^{2}$ Secondly, there is some evidence to suggest that methanogenic persons may suffer from less 'gas' symptoms. ' ${ }^{3} \mathrm{Gas}$ ' is a major symptom of a subset of irritable bowel sufferers. It is also a cause of the pain or discomfort associated with organic disease, such as ulcerative colitis. This symptom may be reduced in methanogenic persons because the oxidation of hydrogen gas to methane gas reduces the volume of gas fourfold. Moreover, pulmonary excretion of methane may also be less limited by intestinal blood flow compared with hydrogen because methane is more soluble than hydrogen.

Previous explanations for the epidemiology of methanogenesis have not provided satisfactory reasons for observed differences between or within populations.

We have speculated that an essential difference between the human colon and other anaerobic environments (such as the rumen) with respect to methanogenesis is the presence of bile acid, which is lost into the colon from the small intestine. ${ }^{4}$ We have previously shown that bile acid inhibits methanogenesis by in vitro human faecal cultures. This inhibition was blocked by the addition of the bile acid chelating agent, cholestyramine. ${ }^{4}$ We now report a similar in vitro inhibition by human bile. This inhibition occurs in vitro at total bile acid concentrations as low as $0.02 \mathrm{mmol} / \mathrm{l}$. We also report the in vivo effect of bile diversion on breath methane excretion in a choledochostomy patient.

\section{Methods}

Human bile was collected from three cholecystectomised patients with bile duct fistulas. Two of these patients had methanogenic faeces (headspace methane in $10 \%$ faecal cultures buffered with HEPES $>4000 \mathrm{ppm}$ at four hours) and the third patient was 'nonmethanogenic' (headspace methane $<30 \mathrm{ppm}$ at four hours). The biles were adjusted to $\mathrm{pH} 7.5$ by addition of $2 \mathrm{~N} \mathrm{HCl}$, and added to serum tubes containing modified Balch No 1 media. ${ }^{56}$ The final bile concentrations in each of the serum tubes was $0,0.3$, $3.0,10$, and $30 \%(\mathrm{v} / \mathrm{v})$; the concentration of Balch media was constant. Faeces, collected from five healthy methanogenic human volunteers, were homogenised in a stomacher, strained through $0.5 \mathrm{~mm}$ cotton gauze, and then added to the bile amended Balch media in the serum tubes to make $0 \cdot 1$ g/\% faecal suspensions. Experiments were performed for each subject using all biles once over a four month period. The serum tubes were stopped with butyl rubber caps (Wheaton), and their headspaces evacuated with oxygen free $\mathrm{H}_{2} / \mathrm{CO}_{2}(80 \% / 20 \%)$ gas under pressure. The choledochostomy patients did not have antibiotics for the 72 hours before or during the bile collection. None of the healthy methanogenic volunteers were ingesting or had ingested antibiotics during the antecedant two months of the study. Cholestyramine $(0.6 \mathrm{~g} / \%)$ was added to some control and bile amended serum tubes. 


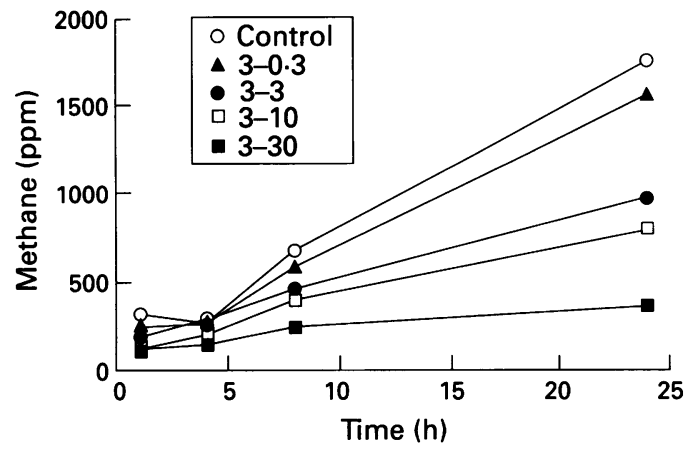

Figure 1: Headspace methane concentrations at different times: an experiment using $0 \cdot 1 \%$ faecal suspensions from one subject and human liver bile no 3 (concentration range $0 \cdot 3-30 \%$ ).

Gas was sampled by syringe needle using a sterile technique from duplicate cultures at each bile concentration from each methanogenic subject at two to four, six to eight, and 24 hours. Methane was measured by gas chromatography with a flame ionisation detector (Packard GC model 428 with a $4 \mathrm{~mm} \times 2 \mathrm{~m}$ glass column packed with Poropak $Q$, carrier gas was nitrogen, injection temperature was $25^{\circ} \mathrm{C}$, oven temperature $50^{\circ} \mathrm{C}$, and detector temperature $200^{\circ} \mathrm{C}$ ). The instrument was calibrated with an argon gas standard that contained $57 \mathrm{ppm}$ methane. Peak heights were linear for methane over the range of $0-100$ ppm. Higher concentrations were measured by diluting the samples with air, and making the appropriate calculations for the deadspace losses with each dilution. Up to four dilutions were required for some gas samples. Ambient methane was subtracted from measurements on diluted samples. The detection limit for methane was $<1$ ppm. Preliminary experiments with one subject ( $\mathrm{n}=3$ faecal collections over two months) had established the reproducibility of methane measurement in the headspace of the in vitro bile amended cultures. The coefficient of variation of per cent methane concentration at four hours incubation was 12 per cent.

End expiratory breath was collected from a fourth choledochostomy patient who had not been treated with antibiotics, for measurement of methane in breath at various times up to 40 weeks after closure of the choledochostomy.

Per cent methane concentration - methane concentration in the headspace was expressed as a value relative to the methane concentration in the headspace of a corresponding control (no bile) culture. This was done by dividing all gas concentration measurements at a given time by the corresponding methane concentration in the headspace of the control culture and multiplying by 100 .

Total bile acids were measured colorimetrically by a commercially available enzymatic assay kit (Randox, Antrim, UK), which measures all bile acids except 3-sulphobile acids.

The cholestyramine (Questran) was a gift of Astra Pharmaceuticals (Australia). Statistical differences were determined by the paired two sided $t$ test for paired data relating bile concentration with methane, and 2 factor analysis of

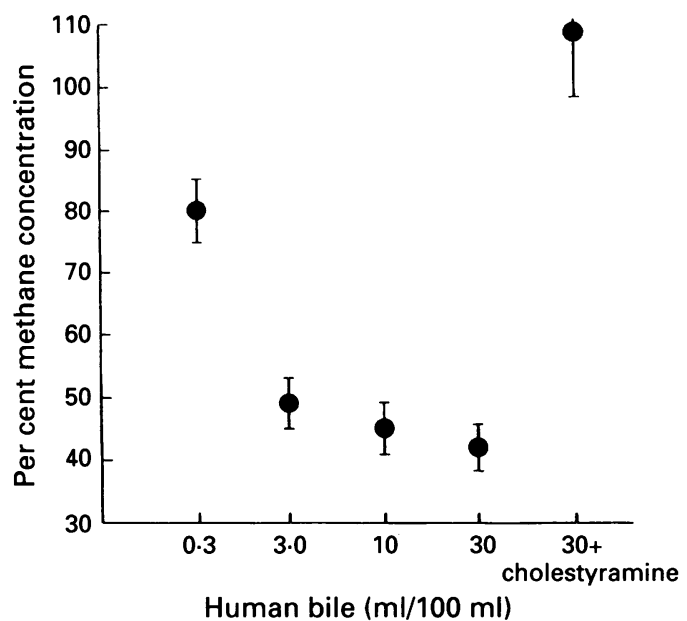

Figure 2: Human bile concentration versus per cent methane concentration: pooled data from all subjects and all headspace sampling times (two to four, six to eight, and 24 hours). There was a dose dependent inhibition of methanogenesis, which was significant at the lowest bile concentration $(0.3 \%)$ tested, and which plateaued at human liver bile concentrations $\geqslant 10 \%$. The inhibition was overcome by the addition of $0.6 \mathrm{~g} / \%$ cholestyramine.

variance for 'between subject' and 'between bile' variations in headspace methane (StatView). Results were deemed significant if $\mathrm{p}$ was less than $0 \cdot 05$. The project was approved by the Mater Adult Hospital ethics committee.

\section{Results}

Methane increased with time in both bile free and bile $(0.3-30 \%)$ amended methanogenic cultures. Figure 1 shows headspace methane concentrations at different times for a typical experiment in which faecal suspensions from one subject were cultured in different concentrations of a human bile (No 3 ).

Human biles inhibited methanogenesis in faecal Balch cultures from all subjects. Inhibition by bile was statistically significant at concentrations of human bile $\geqslant 3.0 \%$ at two to four hours, $\geqslant 3.0 \%$ at eight hours, and $\geqslant 0.3 \%$ at 24 hours. The inhibition was dose related with maximum inhibition plateauing at bile concentrations greater than or equal to $10 \%$. Maximum inhibition by bile, median (range), was $38(0.9-56) \%$ of control methane concentration. 'Between subject' and 'between bile' variations in maximum inhibition of headspace methane were not statistically significant.

Figure 2 shows the relation between human bile concentration and per cent methane concentration. Each point is the mean (SEM) of pooled data from five experiments involving all subjects $(n=5)$, all biles $(n=3)$, and all headspace sampling times (two to four, six to eight, and 24 hours). Thus, each of the points represents the mean (SEM) of 45 measurements. Inhibition was significant at the lowest human liver bile concentration, headspace methane concentration being $80(5 \cdot 2) \%$ of control headspace methane at $0.3 \%$ bile concentration, 49 $(4.0) \%$ of control methane at a $3 \%$ bile concentration, $45(4 \cdot 1) \%$ of control methane at $10 \%$ bile concentration, and $42(3.7) \%$ of control methane at a human bile concentration of $30 \%$ 


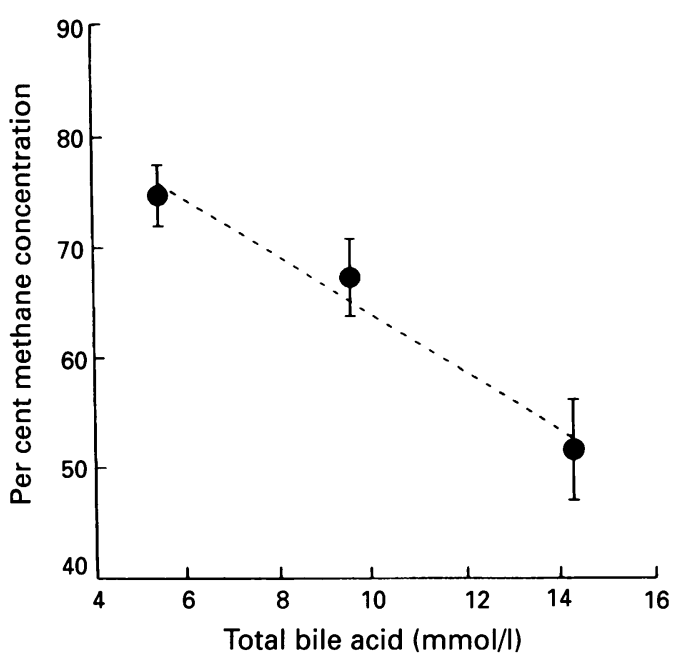

Figure 3: Total bile acid concentration in three human biles versus per cent methane concentration in the headspace of in vitro cultures, showing an inverse linear relation between total bile acid concentration in human liver bile and in vitro methanogenesis (regression coefficient $=0.98$ ).

in the in vitro cultures. The inhibition was overcome by the addition of $0.6 \mathrm{~g} / \%$ cholestyramine (Fig 2), which resulted in headspace methane of $108(10 \cdot 2) \%$, similar to that of the control and the 'control+cholestyramine' cultures, 98 $(9 \cdot 9) \%$.

Figure 3 shows the relation between total bile acid concentration in the biles and the inhibitory effect of the three human biles on the in vitro cultures. Data were pooled from all five subjects and from each of the four concentrations of the bile amended cultures at eight hours $(n=20)$. Thus, each point represents per cent methane concentration in the headspace, mean (SEM), for a human liver bile, versus concentration of bile acid in that bile. There was an inverse linear relation between total bile acid concentration in the human bile and methanogenesis. The bile with the highest concentration of bile acid came from the patient who had 'non-methanogenic' faeces; the other two patients had methanogenic faeces.

Breath methane in a fourth cholecystectomised patient with choledochostomy, was measured immediately before reversal of a 20 day old choledochostomy ( 0 weeks) and then at 4,24 , and 40 weeks after correction of the bile fistula. The breath methanes were respectively $79,29,1$, and $1 \mathrm{ppm}$ above ambient values. The bile from this patient caused a profound dose dependent inhibition of methanogenesis in faecal cultures of three subjects tested. The maximum inhibition by this bile, mean (SEM), was $3.3(0.95) \%$ of control methane at $30 \%$ bile concentration.

\section{Discussion}

Human liver bile inhibited methanogenesis at all concentrations tested $(0 \cdot 3-30 \% \mathrm{v} / \mathrm{v})$, and this effect was reversed by cholestyramine.

The obvious question that arises, is, how relevant are the in vitro experiments to the in vivo situation? The maximum inhibition of methanogenesis by the three human biles, median (range), was $38(0.9-56) \%$ of control headspace methane concentration. Thus at first sight, the inhibitory effect of human bile on in vitro methanogenesis does not explain the five $\log$ concentration difference in the number of methanogens between methanogenic and 'non-methanogenic' faeces. ${ }^{7}$ The in vitro experiments used Balch media. This medium, which was designed for the express purpose of supporting the growth of methanogens is, as with the contents of the caecum, not substrate limited. The in vitro experiments were batch cultures, however, there being no addition of substrate or fresh media after the start of incubation. In this important respect, they did not mimic normal caecal physiology, which behaves as a semi-continuous culture in which substrate and fresh media are added and metabolic products are removed throughout incubation. Such considerations might explain the quantitative difference between the in vitro experimental findings of this study and the numbers of methanogens in methanogenic and 'non-methanogenic' faeces.

The concentrations of total bile acid at the start of incubation in the $0 \cdot 3,3 \cdot 0,10,30 \%$ in vitro cultures amended with bile from the 'non-methanogenic' choledochostomy patient, are calculated at $0.04,0.43,1.4$, and $4.3 \mathrm{mmol} / 1$ respectively. These values should be compared with bile acid concentrations in caecal contents of healthy humans, which are $0 \cdot 1-1 \cdot 0 \mathrm{mmol} / 1 .{ }^{89}$ Using calculations based on the data of Pochart et $a l,{ }^{7}$ the concentrations of methanogens and total anaerobic bacteria at the start of incubation of the in vitro $0.1 \%$ faecal cultures were $\log 4.3$ and $\log 8 \cdot 2 / \mathrm{ml}$ respectively. These values are comparable to the concentrations of methanogens and anaerobic bacteria in the caecal contents of normal humans, which are for methanogens, $\log 2 \cdot 6-5 \cdot 9 / g$ wet weight, and for total anaerobes, $\log 7 \cdot 8-8 \cdot 9 / \mathrm{g}$ wet weight. ${ }^{7}$ Thus, in these respects, the in vitro experiments mimicked normal caecal physiology.

Thus although not conclusive, the in vitro data support the contention that bile acid losses into the caecum are a controlling factor in methanogenesis. Inhibition of methanogens in the caecum by bile acid, and comparatively fast colonic transit time could significantly reduce the total number of methanogens in the colon. This would explain much of the known epidemiology of 'non-methanogenesis' in humans, ${ }^{4}$ which has been related to obesity, ${ }^{10}$ (comparatively) fast colonic transit in healthy persons, ${ }^{11}$ and to small intestinal Crohn's disease. ${ }^{12-14}$

Furthermore, the breath methane excretion data from a choledochostomy patient, provide in vivo evidence in support of the hypothesis that bile acid losses into the caecum determine methanogenesis. After reversal of a bile fistula, the patient was converted in just three months from a highly methanogenic status to a 'nonmethanogenic' status.

Other aspects of bile acid metabolism may also be important, including individual bile acid concentrations in bile and in caecal contents, ileal absorptive capacity, ${ }^{15}$ bacterial degradation of bile acid, and methanogen species. Methanogenesis is one of several 
routes for bacterial disposal of hydrogen gas, which are potentially disrupted by bile acid. ${ }^{4}$ Future research in this field could clarify possible pathogenic relations between colonic bacteria, dietary substrate, and bile. This may have particular relevance to the subset of patients whose main symptom complaint is abdominal distension.

The authors wish to thank the Mater Misericordiae Hospital J P Kelly Research Foundation for their support. They also wish to acknowledge D M Cowley and S A Moody for their helpful discussion and contribution to the assay of bile acids. The authors thank Astra Pharmaceuticals Pty Ltd (Australia) for the gift of Questran. The work has been presented in part to the American in Gastroenterology 1994; 106: A1756, and to the Gastroenterology Society of Australia, $\mathcal{F}$ Gastroenterol Hepatol 1994; 9: A56.

1 Thauer $\mathrm{R}$. Limitation of microbial $\mathrm{H}_{2}$ formation via fermenJ, eds. Microbial energy conversion. Gottingen: Erich Golt KG, 1976: 201-4.

2 Segal I, Walker ARP, Lord S, Cummings JH. Breath methane and large bowel cancer risk in contrasting African populations. Gut 1988; 29: 608-13.

3 Fitzgerald JA, Kajs TM, Buckner RY, Wojtowicz GA, Morel JG, Stinson BS, et al. Comparison of gas production and symptomatic response of methanogenic versus nonmethanogenic subjects to non-absorbable carbononmethanogenic subjects to non-abso

4 Florin THJ, Jabbar IA. A possible role for bile in the control of methanogenesis and accumulation of hydrogen gas in the human colon. $\mathcal{F}$ Gastroenterol Hepatol 1994; 9: 112-7. 5 Balch WE, Fox GE, Woese CR, Wolfe RS. Methanogens: reevaluation of a unique biological group. Microbiol Rev 1979; 43: 260-96.

6 Whitman WB, Bowen TL, Boone DR. The methanogenic bacteria. In: Balows A, Truper HG, Dworkin M, Harder W, Schleiffer KH, eds. The prokaryocytes. 2nd ed. New York: Springer-Verlag, 1992.

7 Pochart P, Lemann F, Flourie B, Pellier P, Goderel I, Rambaud J-C. Pyxigraphic sampling to enumerate methanogens and anaerobes in the right colon of healthy humans. Gastroenterology 1993; 105: 1281-5.

8 McLeod GM, Wiggins HS. Bile-salts in small intestinal contents after ileal resection and in other malabsorption syndromes. Lancet 1968; i: 873-6.

9 Percy-Robb IW, Jalan KN, McManus PA, Sircus W. Effect of ileal resection on bile salt metabolism in patients with ileostomy following proctocolectomy. Clin Sci 1970; 41: ileostomy

10 Haines AP, Imeson JD, Wiggins HS. Relation of breath methane with obesity and other factors. Int $\mathcal{F}$ Obesity 1984; 8: $675-80$.

11 Stephen AM, Wiggins HS, Englyst HN, Cole TJ, Wayman $\mathrm{BJ}$, Cummings JH. The effect of age, sex and level of intake of dietary fibre from wheat on large bowel function in thirty healthy subjects. Br f Nutr 1986; 56: 349-61.

12 Bjørneklett A, Fausa O, Midtvedt T. Bacterial overgrowth in jejunal and ileal disease. Scand 7 Gastroenterol 1983; 18: 289-98.

13 McKay LF, Eastwood MA, Brydon WG. Methane excretion in man - a study of breath, flatus and faeces. Gut 1985; 26: 69-74.

14 Peled Y, Weinberg D, Hallak A, Gilat T. Factors affecting methane production in humans. Dig Dis Sci 1987; 32: methane

15 Florin THJ, Leggett F, Thomas BJ. Intestinal bile acid losses may control methanogenesis. Gastroenterology 1994; 106: A1755. 